\title{
Structure of the Texas Orocline beneath the sedimentary cover (southeast Queensland, Australia)
}

\section{S. BROOKE-BARNETT ${ }^{1,2}$ AND G. ROSENBAUM ${ }^{1}$}

${ }^{1}$ School of Earth Sciences, The University of Queensland, Brisbane 4072, Australia ${ }^{2}$ Origin Energy, 135 Coronation Drive, Milton 4064, Australia

Corresponding author: Samuel Brooke-Barnett

Ph: +61 450092811

Email: Samuel.brooke-barnett@originenergy.com.au

\section{SUPPLEMENTARY PAPERS}

Australian Journal of Earth Sciences (2015) 62, http://dx.doi.org/10.1080/08120099.2015.1058293

Copies of Supplementary Papers may be obtained from the Geological Society of Australia's website (www.gsa.org.au), the Australian Journal of Earth Scienecs website (www.ajes.com.au) or from the National Library of Australia's Pandora archive (http://nla.gov.au/nla.arc-25194).

\section{SUPPLEMENTARY PAPER}

Table: Names and location data for wells that intersect basement in the Surat and Clarence-Moreton basins. 
Table: Names and location data for wells that intersect basement in the Surat and Clarence-Moreton basins.

\begin{tabular}{|c|c|c|c|c|c|c|c|}
\hline \multirow[t]{2}{*}{ Well } & \multicolumn{5}{|c|}{ Location Data } & \multirow{2}{*}{$\begin{array}{c}\text { Basement depth } \\
\text { corrected to } 244 \\
\text { m asl }\end{array}$} & \multirow[t]{2}{*}{ Basement Lithology } \\
\hline & Easting & Northing & $\begin{array}{l}\text { Zone } \\
\text { (UTM) }\end{array}$ & Latitude & Longitude & & \\
\hline ABERCORN 1 & 319341 & 7219424 & $\overline{56 J}$ & -25.1303030 & 151.2079940 & 2082.2 & Camboon Volcanics \\
\hline ABERDEEN PARK 1 & 275729 & 6967977 & $56 \mathrm{~J}$ & -27.3931389 & 150.7319472 & 1125.4 & Combarngo Volcanics \\
\hline ALICK CREEK 1 & 255787 & 6976424 & $56 \mathrm{~J}$ & -27.3135278 & 150.5321111 & 2697.0 & Kuttung Formation \\
\hline BAYLAM 1 & 437512 & 6959531 & $56 \mathrm{~J}$ & -27.4863940 & 152.3674430 & 981.0 & Undifferentiated Basement \\
\hline BENDIDEE 1 & 235939 & 6865148 & $56 \mathrm{~J}$ & -28.3132897 & 150.3070562 & 1232.0 & Kuttung Formation \\
\hline BINKEY 1 & 226319 & 7082647 & $56 \mathrm{~J}$ & -26.3500000 & 150.2577778 & 563.4 & Kuttung Formation \\
\hline BINKEY BAY 1 & 225817 & 7074958 & $56 \mathrm{~J}$ & -26.4192494 & 150.2511064 & 684.9 & Kuttung Formation \\
\hline BOOBERANNA 1 & 187533 & 6847318 & $56 \mathrm{~J}$ & -28.4633600 & 149.8091690 & 2332.5 & Kuttung Formation \\
\hline BRAEMAR 1 & 272462 & 6979658 & $56 \mathrm{~J}$ & -27.2872222 & 150.7011111 & 1296.8 & Kuttung Formation \\
\hline BRIGALOW CREEK 1 & 237265 & 6942519 & $56 \mathrm{~J}$ & -27.6158570 & 150.3377600 & 1625.7 & Undifferentiated Basement \\
\hline BULLOCK CREEK 1 & 234106 & 7077633 & $56 \mathrm{~J}$ & -26.3966940 & 150.3346900 & 465.3 & Kuttung Formation \\
\hline BURUNGA 1 & 207661 & 7121224 & $56 \mathrm{~J}$ & -25.9984200 & 150.0797010 & 2971.7 & Cracow Formation \\
\hline CABAWIN 1 & 222423 & 6955645 & $56 \mathrm{~J}$ & -27.4945333 & 150.1905222 & 3420.0 & Cracow Formation \\
\hline CAMARA 1 & 231088 & 6903286 & $56 \mathrm{~J}$ & -27.9684171 & 150.2663836 & 1557.7 & Kuttung Formation \\
\hline CAMEBY 1 & 228152 & 7047389 & $56 \mathrm{~J}$ & -26.6683333 & 150.2686111 & 1077.6 & Undifferentiated Basement \\
\hline CANAAN 1 & 272098 & 7071459 & $56 \mathrm{~J}$ & -26.4589790 & 150.7141560 & 471.7 & Camboon Volcanics \\
\hline CECIL PLAINS WEST 1 & 318278 & 6952216 & $56 \mathrm{~J}$ & -27.5416830 & 151.1596880 & 1003.4 & Undifferentiated Basement \\
\hline COCKATOO CREEK 1 & 209215 & 7169277 & $56 \mathrm{~J}$ & -25.5653650 & 150.1057520 & 3564.5 & Cracow Formation \\
\hline COMMORON 1 & 251008 & 6846800 & $56 \mathrm{~J}$ & -28.4816860 & 150.4566570 & 762.6 & Undifferentiated Basement \\
\hline CROWDER 1 & 230177 & 6913441 & $56 \mathrm{~J}$ & -27.8766667 & 150.2594444 & 1610.7 & Kuttung Formation \\
\hline CURRAJONG 1 & 226925 & 6908344 & $56 \mathrm{~J}$ & -27.9219680 & 150.2252660 & 1768.2 & Kuttung Formation \\
\hline DEEP CROSSING 1 & 251767 & 6959300 & $56 \mathrm{~J}$ & -27.4672450 & 150.4880330 & 1571.3 & Undifferentiated Basement \\
\hline DEVONDALE 1 & 259799 & 7016769 & $56 \mathrm{~J}$ & -26.9503133 & 150.5804840 & 1014.8 & Undifferentiated Basement \\
\hline DILBONG 1 & 241218 & 6947624 & $56 \mathrm{~J}$ & -27.5705790 & 150.3788700 & 1615.6 & Kuttung Formation \\
\hline DUNMORE CREEK 1 & 283582 & 6951253 & $56 \mathrm{~J}$ & -27.5452778 & 150.8083333 & 1198.9 & Undifferentiated Basement \\
\hline DURABILLA 1 & 288941 & 6950823 & $56 \mathrm{~J}$ & -27.5500000 & 150.8625000 & 1099.9 & Undifferentiated Basement \\
\hline DURABILLA WEST 1 & 280538 & 6948058 & $56 \mathrm{~J}$ & -27.5736111 & 150.7769444 & 1304.7 & Undifferentiated Basement \\
\hline GREEN SWAMP 1 & 248591 & 6960184 & $56 \mathrm{~J}$ & -27.4586940 & 150.4560980 & 1736.0 & Kuttung Formation \\
\hline
\end{tabular}




\begin{tabular}{|c|c|c|c|c|c|c|c|}
\hline \multirow[t]{2}{*}{ Well } & \multicolumn{5}{|c|}{ Location Data } & \multirow{2}{*}{$\begin{array}{c}\text { Basement depth } \\
\text { corrected to } 244 \\
\text { m asl }\end{array}$} & \multirow[t]{2}{*}{ Basement Lithology } \\
\hline & Easting & Northing & $\begin{array}{l}\text { Zone } \\
\text { (UTM) }\end{array}$ & Latitude & Longitude & & \\
\hline JANDOWAE SOUTH 1 & 300106 & 7031385 & $\overline{56 J}$ & -26.8248050 & 150.9885980 & $\overline{591.2}$ & Undifferentiated Basement \\
\hline JANDOWAE WEST 1 & 302822 & 7042979 & $56 \mathrm{~J}$ & -26.7205760 & 151.0177370 & 436.0 & Undifferentiated Basement \\
\hline KEGGABILLA 1 & 252528 & 6913819 & $56 \mathrm{~J}$ & -27.8775861 & 150.4863472 & 1398.6 & Kuttung Formation \\
\hline KILLALOE 1 & 226793 & 6919000 & $56 \mathrm{~J}$ & -27.8258570 & 150.2263760 & 1677.5 & Kuttung Formation \\
\hline KILLAWARRA 1 & 238011 & 6943583 & $56 \mathrm{~J}$ & -27.6064120 & 150.3455370 & 1712.9 & Kuttung Formation \\
\hline KOGAN 1 & 281727 & 7001932 & $56 \mathrm{~J}$ & -27.0877778 & 150.7986111 & 885.0 & Undifferentiated Basement \\
\hline KOGAN SOUTH 1 & 282195 & 6995660 & $56 \mathrm{~J}$ & -27.1444444 & 150.8022222 & 1017.9 & Undifferentiated Basement \\
\hline KUMBARILLA 1 & 281148 & 6964472 & $56 \mathrm{~J}$ & -27.4256350 & 150.7860920 & 1177.8 & Undifferentiated Basement \\
\hline KUMBARILLA EAST 1 & 286492 & 6973132 & $56 \mathrm{~J}$ & -27.3483530 & 150.8416360 & 1215.1 & Undifferentiated Basement \\
\hline LIDDELL 1 & 239289 & 6945366 & $56 \mathrm{~J}$ & -27.5905790 & 150.3588700 & 1622.9 & Undifferentiated Basement \\
\hline MARMADUA 2 & 264260 & 6965337 & $56 \mathrm{~J}$ & -27.4150210 & 150.6155300 & 1266.9 & Kuttung Formation \\
\hline MAXIMA 1 & 225174 & 6869180 & $56 \mathrm{~J}$ & -28.2747222 & 150.1983333 & 2162.7 & Kuttung Formation \\
\hline MIDDLE CREEK 1 & 234673 & 6939321 & $56 \mathrm{~J}$ & -27.6441900 & 150.3108160 & 1676.6 & Kuttung Formation \\
\hline MILLMERRAN 1 & 327423 & 6926552 & $56 \mathrm{~J}$ & -27.7744590 & 151.2485780 & 436.3 & Undifferentiated Basement \\
\hline MINIMA 1 & 217324 & 6859811 & $56 \mathrm{~J}$ & -28.3575240 & 150.1161060 & 2100.5 & Kuttung Formation \\
\hline MINNABILLA 1 & 230102 & 6908223 & $56 \mathrm{~J}$ & -27.9237028 & 150.2574972 & 1584.1 & Undifferentiated Basement \\
\hline MOOGOON 1 & 259478 & 6859885 & $56 \mathrm{~J}$ & -28.3652778 & 150.5458333 & 895.1 & Undifferentiated Basement \\
\hline MOONIE 1 & 229710 & 6928153 & $56 \mathrm{~J}$ & -27.7439130 & 150.2580410 & 1691.2 & Kuttung Formation \\
\hline MOONIE 12 & 226282 & 6924595 & $56 \mathrm{~J}$ & -27.7753020 & 150.2224860 & 1717.1 & Kuttung Formation \\
\hline MOONIE 14 & 229757 & 6927291 & $56 \mathrm{~J}$ & -27.7516900 & 150.2583180 & 1598.5 & Kuttung Formation \\
\hline MOONIE 15 & 227861 & 6926318 & $56 \mathrm{~J}$ & -27.7600840 & 150.2388820 & 1679.6 & Kuttung Formation \\
\hline MOONIE 16 & 232068 & 6930608 & $56 \mathrm{~J}$ & -27.7222460 & 150.2824840 & 1718.8 & Kuttung Formation \\
\hline MOONIE 21 & 227964 & 6925396 & $56 \mathrm{~J}$ & -27.7684170 & 150.2397150 & 1645.0 & Undifferentiated Basement \\
\hline MOONIE 29 & 229015 & 6927422 & $56 \mathrm{~J}$ & -27.7503620 & 150.2508260 & 1689.4 & Undifferentiated Basement \\
\hline MOONIE 3 & 231237 & 6929727 & $56 \mathrm{~J}$ & -27.7300240 & 150.2738730 & 1690.5 & Kuttung Formation \\
\hline MOONIE 39 & 228836 & 6926895 & $56 \mathrm{~J}$ & -27.7550820 & 150.2488970 & 1772.9 & Undifferentiated Basement \\
\hline MOONIE 6 & 229150 & 6926231 & $56 \mathrm{~J}$ & -27.7611350 & 150.2519300 & 1691.8 & Kuttung Formation \\
\hline MOONIE 7 & 227036 & 6925197 & $56 \mathrm{~J}$ & -27.7700240 & 150.2302640 & 1689.7 & Kuttung Formation \\
\hline MOONIE NORTH 1 & 233493 & 6934367 & $56 \mathrm{~J}$ & -27.6886350 & 150.2977610 & 1694.5 & Undifferentiated Basement \\
\hline
\end{tabular}




\begin{tabular}{|c|c|c|c|c|c|c|c|}
\hline \multirow[t]{2}{*}{ Well } & \multicolumn{5}{|c|}{ Location Data } & \multirow{2}{*}{$\begin{array}{c}\text { Basement depth } \\
\text { corrected to } 244 \\
\text { m asl }\end{array}$} & \multirow[t]{2}{*}{ Basement Lithology } \\
\hline & Easting & Northing & $\begin{array}{l}\text { Zone } \\
\text { (UTM) }\end{array}$ & Latitude & Longitude & & \\
\hline MURILLA 1 & 242824 & 6946950 & $\overline{56 J}$ & -27.5769670 & 150.3949810 & 1674.0 & Kuttung Formation \\
\hline NANGWAY 1 & 298712 & 6925678 & $56 \mathrm{~J}$ & -27.7783500 & 150.9571950 & 1001.4 & Undifferentiated Basement \\
\hline OVERFLOW 1 & 485456 & 6909435 & $56 \mathrm{~J}$ & -27.9399990 & 152.8521610 & 678.6 & Undifferentiated Basement \\
\hline PARINGA 1 & 216942 & 6856446 & $56 \mathrm{~J}$ & -28.3877778 & 150.1113889 & 2006.6 & Undifferentiated Basement \\
\hline PELHAM 1 & 229743 & 7078089 & $56 \mathrm{~J}$ & -26.3917560 & 150.2910970 & 581.1 & Kuttung Formation \\
\hline PIPECLAY 1 & 235281 & 6906590 & $56 \mathrm{~J}$ & -27.9394670 & 150.3097090 & 1533.3 & Kuttung Formation \\
\hline PRING 1 & 224735 & 6917936 & $56 \mathrm{~J}$ & -27.8350240 & 150.2052650 & 1735.7 & Undifferentiated Basement \\
\hline RIVERVIEW 1 & 245513 & 6960189 & $56 \mathrm{~J}$ & -27.4580790 & 150.4249790 & 1862.9 & Undifferentiated Basement \\
\hline ROCK CREEK 1 & 274887 & 6971756 & $56 \mathrm{~J}$ & -27.3589100 & 150.7241390 & 1286.4 & Kuttung Formation \\
\hline ROCKWOOD 1 & 239014 & 7013206 & $56 \mathrm{~J}$ & -26.9786948 & 150.3705388 & 1214.8 & Kuttung Formation \\
\hline ROCKWOOD 2 & 239421 & 7012199 & $56 \mathrm{~J}$ & -26.9878613 & 150.3744277 & 1493.1 & Kuttung Formation \\
\hline ROCKWOOD NORTH 1 & 241527 & 7017108 & $56 \mathrm{~J}$ & -26.9439724 & 150.3966493 & 1174.9 & Kuttung Formation \\
\hline ROPELEY 1 & 428756 & 6941174 & $56 \mathrm{~J}$ & -27.6516720 & 152.2777250 & 573.0 & Buaraba Formation \\
\hline SAWPIT GULLY 1 & 264081 & 6960344 & $56 \mathrm{~J}$ & -27.4600210 & 150.6127530 & 1431.8 & Kuttung Formation \\
\hline SCOTIA 1 & 207641 & 7126835 & $56 \mathrm{~J}$ & -25.9478093 & 150.0807556 & 2915.9 & Camboon Volcanics \\
\hline SCOTIA 2 & 206865 & 7130548 & $56 \mathrm{~J}$ & -25.9141704 & 150.0738388 & 2825.0 & Camboon Volcanics \\
\hline SHINGLE HUT 1 & 226494 & 7088653 & $56 \mathrm{~J}$ & -26.2958620 & 150.2608010 & 480.5 & Camboon Volcanics \\
\hline SPECULATION 1 & 267167 & 7069684 & $56 \mathrm{~J}$ & -26.4741910 & 150.6644070 & 398.8 & Camboon Volcanics \\
\hline STATION CREEK 1 & 301824 & 6924807 & $56 \mathrm{~J}$ & -27.7866667 & 150.9886111 & 839.3 & Undifferentiated Basement \\
\hline STITCH 1 & 232715 & 6896718 & $56 \mathrm{~J}$ & -28.0279722 & 150.2814194 & 1781.0 & Kuttung Formation \\
\hline STOCKYARD CREEK 1 & 279772 & 7081083 & $56 \mathrm{~J}$ & -26.3733570 & 150.7927370 & 345.8 & Undifferentiated Basement \\
\hline STRATHPINE 1 & 224098 & 7077745 & $56 \mathrm{~J}$ & -26.3937830 & 150.2344900 & 714.6 & Kuttung Formation \\
\hline STRATHPINE 2 & 224027 & 7076835 & $56 \mathrm{~J}$ & -26.4019730 & 150.2335810 & 715.0 & Kuttung Formation \\
\hline STRATHPINE 3 & 224224 & 7075392 & $56 \mathrm{~J}$ & -26.4150280 & 150.2352480 & 722.9 & Kuttung Formation \\
\hline SUNNY CORNER 1 & 238639 & 6852254 & $56 \mathrm{~J}$ & -28.4300867 & 150.3316598 & 1045.0 & Kuttung Formation \\
\hline SUSSEX DOWNS 1 & 218176 & 6924473 & $56 \mathrm{~J}$ & -27.7747222 & 150.1402778 & 3195.7 & Kuttung Formation \\
\hline SWAN CREEK 1 & 421065 & 6877487 & $56 \mathrm{~J}$ & -28.2261150 & 152.1955110 & 71.8 & Undifferentiated Basement \\
\hline TARA 1 & 251852 & 6972792 & $56 \mathrm{~J}$ & -27.3455780 & 150.4916430 & 2047.8 & Kuttung Formation \\
\hline THOMPSON 1 & 246239 & 6873777 & $56 \mathrm{~J}$ & -28.2375000 & 150.4138889 & 1068.5 & Kuttung Formation \\
\hline
\end{tabular}




\begin{tabular}{|c|c|c|c|c|c|c|c|}
\hline \multirow[t]{2}{*}{ Well } & \multicolumn{5}{|c|}{ Location Data } & \multirow{2}{*}{$\begin{array}{c}\text { Basement depth } \\
\text { corrected to } 244 \\
\text { m asl }\end{array}$} & \multirow[t]{2}{*}{ Basement Lithology } \\
\hline & Easting & Northing & $\begin{array}{l}\text { Zone } \\
\text { (UTM) }\end{array}$ & Latitude & Longitude & & \\
\hline TINKER CREEK 1 & 282793 & 6927776 & $56 \mathrm{~J}$ & -27.7569444 & 150.7961111 & 1253.8 & Undifferentiated Basement \\
\hline TIPTON 1 & 321664 & 6968458 & $56 \mathrm{~J}$ & -27.3955720 & 151.1963530 & 1125.5 & Undifferentiated Basement \\
\hline TOOBEAH 1 & 194538 & 6857613 & $56 \mathrm{~J}$ & -28.3722222 & 149.8833333 & 1798.2 & Undifferentiated Basement \\
\hline TOORA 1 & 288674 & 6974155 & $56 \mathrm{~J}$ & -27.3394640 & 150.8638580 & 1070.9 & Undifferentiated Basement \\
\hline UNDULLA 1 & 229410 & 6985383 & $56 \mathrm{~J}$ & -27.2277778 & 150.2677778 & 2518.3 & Kuttung Formation \\
\hline URANILLA 1 & 270001 & 6903303 & $56 \mathrm{~J}$ & -27.9755556 & 150.6616667 & 1135.5 & Kuttung Formation \\
\hline WAGGABA 1 & 294517 & 6934261 & $56 \mathrm{~J}$ & -27.7002778 & 150.9161111 & 1129.7 & Undifferentiated Basement \\
\hline WAMBO CREEK 1 & 262823 & 7000325 & $56 \mathrm{~J}$ & -27.0991667 & 150.6077778 & 1309.3 & Kuttung Formation \\
\hline WEST BRAEMAR 1 & 270863 & 6978903 & $56 \mathrm{~J}$ & -27.2937722 & 150.6848222 & 1052.5 & Kuttung Formation \\
\hline WEST DOCKERILL 1 & 230917 & 6917028 & $56 \mathrm{~J}$ & -27.8444680 & 150.2677640 & 1716.5 & Kuttung Formation \\
\hline WIDARA 1 & 232067 & 6902322 & $56 \mathrm{~J}$ & -27.9773059 & 150.2761058 & 1375.4 & Kuttung Formation \\
\hline WIDGEWA 1 & 230714 & 6900474 & $56 \mathrm{~J}$ & -27.9936949 & 150.2619394 & 1784.1 & Undifferentiated Basement \\
\hline WILKIE 1 & 294720 & 6926290 & $56 \mathrm{~J}$ & -27.7722222 & 150.9168056 & 1052.9 & Undifferentiated Basement \\
\hline WARRAWA 1 & 226379 & 6916735 & $56 \mathrm{~J}$ & -27.8461950 & 150.2216610 & 1659.9 & Kuttung Formation \\
\hline WILLOWBE 1 & 229215 & 6909969 & $56 \mathrm{~J}$ & -27.9077778 & 150.2488889 & 1623.2 & Kuttung Formation \\
\hline WYBAR 1 & 233288 & 6906508 & $56 \mathrm{~J}$ & -27.9398038 & 150.2894529 & 1465.9 & Kuttung Formation \\
\hline XYLANE 1 & 236599 & 7009898 & $56 \mathrm{~J}$ & -27.0080810 & 150.3455300 & 928.9 & Kuttung Formation \\
\hline XYLEX 1 & 242456 & 7025284 & $56 \mathrm{~J}$ & -26.8704083 & 150.4076833 & 1229.7 & Kuttung Formation \\
\hline $\mathrm{XYL}-\mathrm{L} 1$ & 235746 & 7009873 & $56 \mathrm{~J}$ & -27.0081390 & 150.3369280 & 1221.8 & Kuttung Formation \\
\hline XYLOIL 1 & 241718 & 7015880 & $56 \mathrm{~J}$ & -26.9550835 & 150.3983160 & 1207.8 & Kuttung Formation \\
\hline XYLOIL SOUTH 1 & 241468 & 7014705 & $56 \mathrm{~J}$ & -26.9656368 & 150.3955535 & 1219.4 & Kuttung Formation \\
\hline XYLOLEUM 1 & 241306 & 7028538 & $56 \mathrm{~J}$ & -26.8408447 & 150.3967871 & 922.8 & Kuttung Formation \\
\hline XYLON 1 & 235365 & 7021446 & $56 \mathrm{~J}$ & -26.9036929 & 150.3355538 & 1055.1 & Kuttung Formation \\
\hline YARRALA 1 & 319386 & 6998718 & $56 \mathrm{~J}$ & -27.1222222 & 151.1777778 & 842.9 & Undifferentiated Basement \\
\hline YARRILL CREEK 1 & 246651 & 6891621 & $56 \mathrm{~J}$ & -28.0766667 & 150.4219444 & 995.0 & Kuttung Formation \\
\hline ZIG ZAG 1 & 296210 & 6913405 & $56 \mathrm{~J}$ & -27.8886897 & 150.9297060 & 651.5 & Undifferentiated Basement \\
\hline
\end{tabular}

